\title{
Potential of temperature-response collagen-genipin sols as a novel submucosal injection material for endoscopic resection
}

\section{다(1)우우}

\author{
Authors \\ Yusaku Takatori ${ }^{1,2,3}$, Toshio Uraoka ${ }^{4}$, Takefumi Narita ${ }^{5}$, Shunji Yunoki ${ }^{5}$, Naohisa Yahagi ${ }^{3}$
}

Institutions

1 Department of Gastroenterology, National Hospital Organization Tokyo Medical Center, Tokyo, Japan

2 Department of Gastroenterology, National Hospital Organization Saitama Hospital, Saitama, Japan

3 Division of Research and Development for Minimally Invasive Treatment, Cancer Center, Keio University School of Medicine, Tokyo, Japan

4 Department of Gastroenterology and Hepatology, Gunma University School of Medicine, Gunma, Japan

5 Biotechnology Group, Tokyo Metropolitan Industrial Technology Research Institute, Tokyo, Japan

submitted 1.9.2018

accepted after revision 14.2.2019

Bibliography

DOI https://doi.org/10.1055/a-0867-9450 |

Endoscopy International Open 2019; 07: E561-E567

(c) Georg Thieme Verlag KG Stuttgart · New York

ISSN 2364-3722

Corresponding author

Toshio Uraoka, MD, PhD, Department of Gastroenterology and Hepatology, Gunma University School of Medicine, 3-39-22, Showa-machi, Maebashi-shi, Gunma, 371-8511, Japan

Fax: +81-27-220-7798

toshi_urao@yahoo.co.jp

\section{ABSTRACT}

Background and study aims We developed a novel submucosal (SM) injection material that contained pepsin-solubilized collagen (PSC), genipin (Ge) and phosphate buffer (PB). The aim of this study was to validate safety and usability of it for endoscopic resection (ER).

Materials and methods In preliminary studies, 1) appropriate warming time and concentration of $\mathrm{Ge}$, and concentration of $\mathrm{NaCl}$ in $\mathrm{PB}, 2$ ) storage modulus of PSC, Ge, and PB mixture (PSC/Ge), and PSC as a mechanical property, 3) histological finding after injection, and histological toxicity of PSC/Ge was evaluated. We injected PSC/Ge, PSC, sodium hyaluronate (SH), dextrose (DW), and normal saline (NS) into SM of resected porcine stomach. We compared mean height of mucosal elevation after immediate injection $(\mathrm{MH})$ and mean retaining rate at 60 minutes (MR) as ex vivo study.

Results Optimal condition of PSC/Ge was Ge $5.5 \mathrm{mMol}$ with 24 hours worming time and $\mathrm{NaCl} 280 \mathrm{mMol}$. PSC/Ge had better mechanical property than PSC. It was efficiently integrated and confined to the SM with acceptable toxicity. $\mathrm{MH}$ of PSC/Ge $(5.1 \pm 0.74 \mathrm{~mm})$ and PSC $(4.8 \pm 0.84 \mathrm{~mm})$ were significantly higher than NS $(3.2 \pm 0.84 \mathrm{~mm})$. MR of PSC/Ge (100 $\pm 0.0 \%)$ was significantly higher than NS (61.7 $\pm 11.2 \%)$, DW (58.3 $\pm 11.8 \%)$ and $\mathrm{SH}(61.8 \pm 8.6 \%)$.

Conclusion PSC/Ge and PSC has potential to be safe and usable for ER. PSC/Ge was better than PSC because of better mechanical property than PSC.

\section{Introduction}

Endoscopic mucosal resection and endoscopic submucosal dissection are becoming widespread and standard techniques as minimally invasive therapies for early-stage gastrointestinal tumors because of progress in performance of endoscopy and devices $[1,2]$.

Normal saline (NS) is commonly used as submucosal injection material in clinical practice because of its low cost and lack of toxicity to human tissue. However, use of NS may necessitate multiple injections during the procedure because it is quickly absorbed by surrounding tissue and unable to maintain mucosal lifting for a long period [3], which may ultimately lead to prolonged operation times and increased risk of perforation. Therefore, some alternative agents have been proposed for use in submucosal injection to shorten procedure time and provide safer procedure outcomes.

In clinical practice, dextrose (DW) and sodium hyaluronate $(\mathrm{SH})$ are also used as submucosal injection material for endoscopic treatment. However, DW is quickly absorbed by surrounding tissue and cannot maintain mucosal lifting for long periods of time, similar to NS [4]. Although SH exhibits good 
viscosity (better than that of NS) and low toxicity, its use in clinical practice is limited because of its high cost. Other materials have been used for submucosal injections, but only a few materials are utilized clinically [3,5-10].

In a previous study, use of pepsin-digested collagen was proposed because its mechanical properties are increased by genipin as a result of genipin-induced crosslinking, and collagen itself presents temperature responsiveness [11]. Collagen maintains fluidity at room temperature, while increasing temperatures cause gelation and eventually result in solid gels [12]. In addition, collagen is able to fix the properties of a gel depending on the $\mathrm{NaCl}$ concentration in phosphate buffer [13]. Therefore, we conducted this study to evaluate the possibility of using collagen sol and genipin as a submucosal injection material for endoscopic therapy.

\section{Material and methods}

This study was conducted in the laboratory of the National Hospital Organization Tokyo Medical Center and Tokyo Metropolitan Industrial Technology Research Institute. All animal tests were approved by the animal ethics committee of both institutions.

\section{Materials}

Pepsin-solubilized collagen (PSC) at $\mathrm{pH} 3$ was diluted in $\mathrm{HCl}(1 \%$ solution of mainly type I collagen from porcine skin, Nipponham, Japan). Acid-solubilized collagen at $\mathrm{pH} 3$ was diluted in $\mathrm{HCl}(0.3 \%$ solution of mainly type I collagen from porcine tendon, Nitta Gelatin, Japan). Genipin (Ge; Wako Pure Chemical Industries, Japan), phosphate buffered saline (PBS) tablets (Sigma-Aldrich, MO), disodium hydrogen phosphate $\left(\mathrm{Na}_{2} \mathrm{HPO}_{4}\right.$; Wako Pure Chemical Industries, Japan), sodium dihydrogen phosphate $\left(\mathrm{NaH}_{2} \mathrm{PO}_{4}\right.$; Wako Pure Chemical Industries, Japan), $\mathrm{NIH} / 3 \mathrm{~T} 3$ fibroblast cells (RIKEN RCB2767), Dulbecco's modified Eagle's medium (DMEM; Sigma-Aldrich, MO), calf serum (CS; MP Biomedicals, Germany), antibiotic-antimycotic (Gibco, NY), Cell Counting Kit-8 (CCK-8; Dojindo Laboratories, Japan), 0.4\% SH (MucoUp, Boston Scientific Japan, Japan), 5\% DW and NS were purchased and used without further purification.

\section{Adequate condition settings for collagen sol for an endoscopic procedure}

As shown in the Introduction, the condition setting factors for collagen sol were the concentration of $\mathrm{NaCl}$ in PBS, warming time of genipin, and concentration of genipin.

\section{Concentration of $\mathrm{NaCl}$}

To determine the $\mathrm{NaCl}$ concentration of PBS, we prepared PSC sol without genipin; $0.5 \%$ neutral PSC sol was prepared by mixing three components: acidic PSC solution, deionized water, and neutral buffers. The components were prepared as follows: $1 \%$ acidic PSC solution was concentrated to $2 \%$ in a rotary evaporator at $29^{\circ} \mathrm{C}$ and stored in aliquots $(2 \mathrm{~g})$ in biological tubes at $4^{\circ} \mathrm{C}$. Phosphate buffers $(\mathrm{pH} 7)$ were designated $\mathrm{PB}[\mathrm{X}-\mathrm{Y}]$, where $X$ is the total concentration of $\mathrm{Na} 2 \mathrm{HPO} 4 / \mathrm{NaH} 2 \mathrm{PO} 4$, and $\mathrm{Y}$ is the $\mathrm{NaCl}$ concentration. The $2 \%$ acidic PSC solution $(2 \mathrm{~g})$, deionized water $(2 \mathrm{~mL})$, and $\mathrm{PB}[100-\mathrm{Y}](\mathrm{Y}: 0-840)(4 \mathrm{~mL})$ were mixed vigorously, producing $0.5 \%$ neutral PSC sol in $\mathrm{PB}[50-\mathrm{Y}]$ (Y: 0 -420) (designated PSC sol).

The PSC sol gelatins that responded to temperature were evaluated using dynamic viscoelastic tests with a rheometer. During oscillation, the temperature was maintained at $23^{\circ} \mathrm{C}$ for 1 minute, then increased to $37^{\circ} \mathrm{C}$ over 30 seconds to trigger gelation, and subsequently maintained at $37^{\circ} \mathrm{C}$ to promote gelation. Changes in the storage $\left(G^{\prime}\right)$ and loss $\left(G^{\prime \prime}\right)$ moduli were registered throughout the test. The gel point was determined as the point at which $G^{\prime}$ and $G^{\prime \prime}$ intersected in their time-course changes. The gelation time at $37^{\circ} \mathrm{C}(\mathrm{Tg} 37)$ was defined as the time to reach the gel point after the set temperature reached $37^{\circ} \mathrm{C} \mathrm{Tg37}$ indicates the gelation rate of the collagen sol that responds to body temperature. We also measured the gelation time of the collagen sols at $30^{\circ} \mathrm{C}(\mathrm{Tg} 30)$ in a manner similar to $\operatorname{Tg} 37$.

To determine the $\mathrm{NaCl}$ concentration and obtain inflection points for collagen fibril formation rates, $\mathrm{Tg} 37$ for various $\mathrm{NaCl}$ concentrations was plotted against Tg30. Finally, we determined the appropriate $\mathrm{NaCl}$ concentration resulting in rapid gelation at $37^{\circ} \mathrm{C}$ and adequate fluidity at $30^{\circ} \mathrm{C}$

\section{Warming time and concentration of genipin}

PSC/Ge sol was prepared in a manner similar to preparation of PSC sol, except that deionized water was substituted with Ge solution. Ge solution was dissolved in $\mathrm{PB}[100-0]$ at a concentration of $22 \mathrm{mM}$ and then warmed in a water bath at $37^{\circ} \mathrm{C}$ for 12 hours, 24 hours, or 48 hours (designated Ge-12, Ge-24, and Ge-48).

A portion of the Ge solution was diluted from $22 \mathrm{mM}$ to 11 $\mathrm{mM}$ and $5.5 \mathrm{mM}$ with $\mathrm{PB}[100-0]$. A Ge solution in $\mathrm{PB}[100-\mathrm{Y}]$ without warming (designated Ge-0) was also prepared and similarly diluted. The $2 \%$ acidic PSC solution $(2 \mathrm{~g})$, each of the Ge solutions at 5.5-22 mM in PB[100-0] (2mL), and PB[50560] $(4 \mathrm{~mL})$ were mixed vigorously, and $0.5 \%$ neutral PSC/Ge sols in PB[50-280] containing 1.4-5.5 mM Ge-0, Ge-12, Ge24 , or Ge-48 were produced (designated PSC/Ge or PSC/Ge-x sols, where Ge indicates unspecified Ge-x).

PSC/Ge sol gelation was evaluated using dynamic viscoelastic tests with a rheometer, in a manner similar to the study of concentration of $\mathrm{NaCL}$. First, we produced gelation curves for the PSC/Ge sols in relation to the warming time. We fixed the concentration of Ge at $5.5 \mathrm{mM}$. The sharpness of the curves was quantified by $\mathrm{Tg} 37$, which was plotted against the Ge concentration for each Ge sample. Second, the Tg37 for all conditions of the PSC/Ge sol was plotted against $\mathrm{Tg} 30$, and we finally determined the warming time and concentration of genipin needed to accelerate the gelation of PSC within approximately 1 minute.

\section{Rheological evaluations of materials}

To evaluate gelation speed and physical strength response to temperature, we evaluated the viscosity of submucosal injection materials, including PSC/Ge sol, PS sol, SH, and DW, using the dynamic viscoelastic test. During oscillation, the temperature was maintained at $23^{\circ} \mathrm{C}$ for 30 seconds, then increased to 
$37^{\circ} \mathrm{C}$ over a timespan of 30 seconds to trigger gelation, and subsequently maintained at $37^{\circ} \mathrm{C}$. Change in complex viscosity $\left(\eta^{*}\right)$ was registered throughout the test. The submucosal injection material NS exhibited too low a viscosity to allow measurement of $\eta^{*}$ with a rheometer; therefore, it was calculated using SEDNTERP (free software).

\section{Pathological examination}

We performed an in vitro examination to evaluate whether the collagen gel integrated with the submucosa. A 2-mL aliquot of $\mathrm{PSC} / \mathrm{Ge}$ sol was injected into the submucosa of the porcine stomach in a live animal under general anesthesia. Fifteen minutes after injection, the swine was sacrificed, and we excised its stomach. We produced histologic sections from each block, stained them with hematoxylin-eosin (HE) and silver impregnation staining, and observed the submucosa under a microscope via high-magnification viewing.

\section{Evaluation of cytotoxicity}

Cell viability in PSC/Ge sol was tested to evaluate the cytotoxicity of the cell culture system using 24-well cell culture insert plates (IPs), in which the cells and sols were separated during cell culture.

To evaluate the cytotoxicity of cells that were shielded by the submucosa, a modified cell culture system was set up using collagen fibrillar gels to mimic the submucosa and employed for evaluation. A mixture of acid-solubilized collagen, concentrated PBS, and HEPES buffer, which contained $\mathrm{NaOH}, \mathrm{NaHCO}$, and HEPES, was dispensed into the IPs to mimic the submucosa, followed by incubation at $37^{\circ} \mathrm{C}$ for 30 minutes to form collagen gels. NIH-3T3 cell suspensions were spread onto the collagen gel-containing IPs (CIPs) to achieve a cell density of $1.1 \times 10^{5}$ cells $/ \mathrm{cm}^{2}$. Simultaneously, PSC/Ge sol was individually poured into 24 -well culture dishes $(0.5 \mathrm{~g} /$ well) under conditions determined in prior studies and warmed in a water bath at $37^{\circ} \mathrm{C}$ for 15 minutes or 10 minutes.

CIPs were plated in 24-well culture dishes containing PSC sol or PSC/Ge sol or in empty 24-well culture dishes as control cells; $0.2 \mathrm{~mL}$ or $1.4 \mathrm{~mL}$ of DMEM was added to the upper IPs or the bottom sols, respectively, in the well plates. The plates were then incubated at $37^{\circ} \mathrm{C}$ for 24 hours.

The working solution containing CCK-8 in culture medium was added to both the upper insert plates and the bottom well plates. The absorbance of each well was measured at $450 \mathrm{~nm}$ using a microplate reader. The cell viability was calculated using the equation:

Cell viability $(\%)=($ Ace $/ A c) \times 100$

where Ace is the absorbance of the cells after the 24-hour exposure test, and Ac is the absorbance of control cells. Cell viability data are expressed as the mean \pm SD.

\section{Comparison of mean height and retention rate of submucosal elevation}

We cut the upper third of resected porcine stomachs, which are reported to be similar to the human stomach, into approximately $5 \times 5-\mathrm{cm}$ square specimens and stretched them on a rubber board. Porcine stomachs were used within 2 hours of re- section. One milliliter of each of the five solutions (PSC/Ge sol, PSC sol, SH, DW, and NS) was injected diagonally, as in practical endoscopic therapy, into the submucosa of the specimen using a 10.0-mL syringe and an endoscopic needle catheter (Fine Jet S2816, Top Co., Tokyo, Japan). The syringe was extruded using a mechanical syringe pump (KDS100, KD Scientific, United States) at $150 \mathrm{~mL}$ per hour. The needle catheter was heated to $30{ }^{\circ} \mathrm{C}$ to stimulate entry into the endoscopic channel using a water bath, and the porcine specimens were maintained at $37^{\circ} \mathrm{C}$ (human body temperature) using a digital egg incubator (Rcom Max 20, Autoelex Co., Kyungsangnam-Do, Korea). This procedure was carried out in each group five times. We observed and subsequently recorded the height of the whole thickness of the stomach wall as mucosal elevation from the lateral direction using a measuring device immediately after and 15, 30, 45, and 60 minutes after injection. To equalize the observation settings, we photographed the subjects laterally at the same distance, and a ruler was set vertically beside the rubber plates. We compared mean heights of mucosal elevation immediately after injection as well as mean retention rates of mucosal elevation at each time after injection for the five solutions. Mean retention rate was calculated using the equation:

Retention rate $(\%)=($ mucosal elevation of each timing/mucosal elevation after immediately injection) $\times 100$

\section{Statistical analysis}

Statistical analysis was performed using statistical software (JMP, SAS Institute Inc, United States). Outcomes of the study of adequate condition settings for collagen sol for an endoscopic procedure and the comparison of mean height and retention rate of submucosal elevation were analyzed using the Tukey-Kramer test. A $P$ value $<0.05$ was considered to be statistically significant.

\section{Results}

\section{Concentration of $\mathrm{NaCl}$}

- Fig. 1 shows Tg37 plotted against Tg30. Tg37 for $280 \mathrm{mMol}$ $\mathrm{NaCl}$ in $\mathrm{PB}$ was faster than that for the other concentration. The result for $\operatorname{Tg} 30$ was greater than 10 minutes. Finally, we determined that the optimal concentration of $\mathrm{NaCl}$ in $\mathrm{PB}$ was $280 \mathrm{mMol}$ (Tg37: $0.96 \mathrm{~min}, \operatorname{Tg} 30: 13.3 \mathrm{~min})$.

\section{Warming time and concentration of genipin}

Tg37 decreased with increasing Ge concentration in all the PSC/ Ge sols. The Tg37 of PSC sol in the absence of Ge was 3.5 minutes and decreased to 2.67 minutes as the Ge- 0 concentration increased from 0 to $5.5 \mathrm{mM}$. The $\mathrm{Tg} 37$ of the sols containing $5.5 \mathrm{mM}$ Ge decreased from 2.67 minutes to 0.79 minutes as the warming time of Ge increased from 0 to 48 hours. As a result, the Ge concentration-dependent decrease in $\mathrm{Tg} 37$ became steeper as the warming time of Ge increased ( $\$$ Fig. 2a). Tg37 monotonously increased as $\mathrm{Tg} 30$ increased and almost reached a plateau when $\mathrm{Tg} 30>12$ minutes ( $\boldsymbol{\nabla} \mathbf{F i g . 2 b}$ ). According to these results, we determined that the warming time of genipin was 24 hours, and we chose $2.8 \mathrm{mMol}$ as the genipin concentration. 


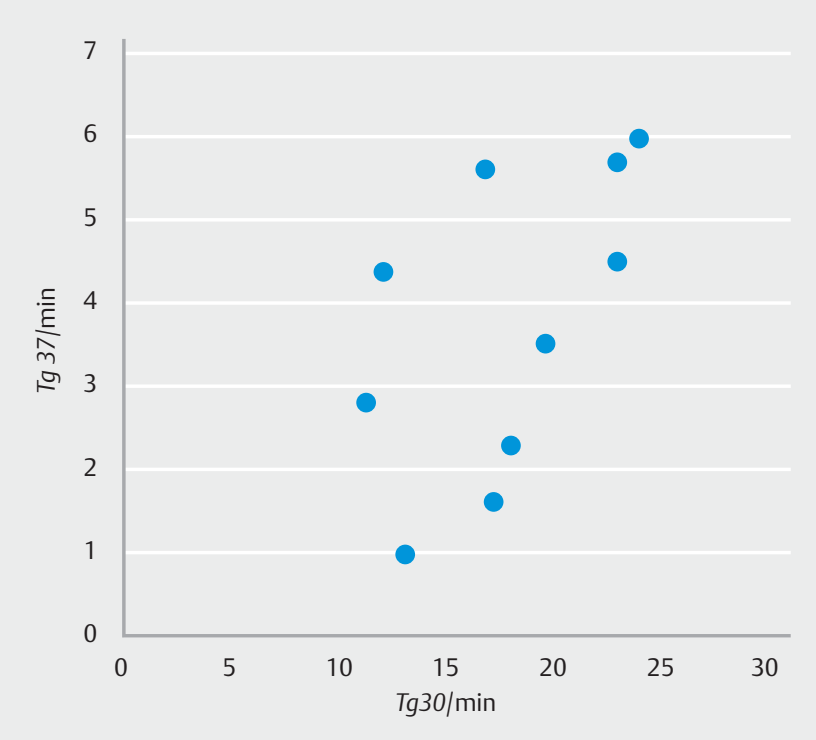

Fig. 1 Plots of $T g 37$ against $T g 30$.

\section{Rheological evaluations of materials}

Fig. 3 shows the change in viscosity of the submucosal injection materials as they respond to increases in temperature from $23^{\circ} \mathrm{C}$ to $37^{\circ} \mathrm{C}$. The $\eta^{*}$ of the PSC and PSC/Ge sols increased exponentially, responding to the increase in temperature, and the $\eta^{*}$ of PSC/Ge was higher than that of PSC at 10 minutes after the temperature reached $37^{\circ} \mathrm{C}$. In contrast, the $\eta^{*}$ of $\mathrm{SH}$ and DW did not show any change, mostly because they do not exhibit temperature responsiveness.

\section{Pathological examination}

Although collagen gel was observed in the submucosa, evaluating whether it integrated with the submucosa via HE staining alone was difficult. However, we clearly observed collagen gel between the reticular fibers (surrounded by yellow circles in submucosa $>$ Fig. $\mathbf{3 b}$ and $\mathbf{F i g . 3 d}$ ) in the using silver impregnation staining. However, the gel did not extend vertically to the submucosa ( $>$ Fig. $4 a-d)$.

\section{Evaluation of cytotoxicity}

- Fig. 5 shows results of the cell viability tests using two types of cell culture systems. The cells cultured in the CIP-PSC system and the CIP-PSC/Ge system showed viabilities of $106 \pm 6 \%$ and $90.4 \pm 5.9 \%$, respectively. The viability in the CIP-PSC/Ge system was significantly lower than that in the CIP-PSC system ( $P$ $<0.01)$.

\section{Comparison of mean height and retention rate of submucosal elevation}

Mean heights of mucosal elevation of PSC/Ge sol $(5.1 \pm$ $0.74 \mathrm{~mm})$, PSC sol $(4.8 \pm 0.84 \mathrm{~mm})$, and SH $(4.8 \pm 0.76 \mathrm{~mm}) \mathrm{im}-$ mediately after injection were significantly higher than that of NS $(3.2 \pm 0.84 \mathrm{~mm})(P<0.05)$ ( $>$ Fig. 6a). Mean retention rate of mucosal elevation for PSC/Ge sol (100 $\pm 0 \%$ ) was shown to be

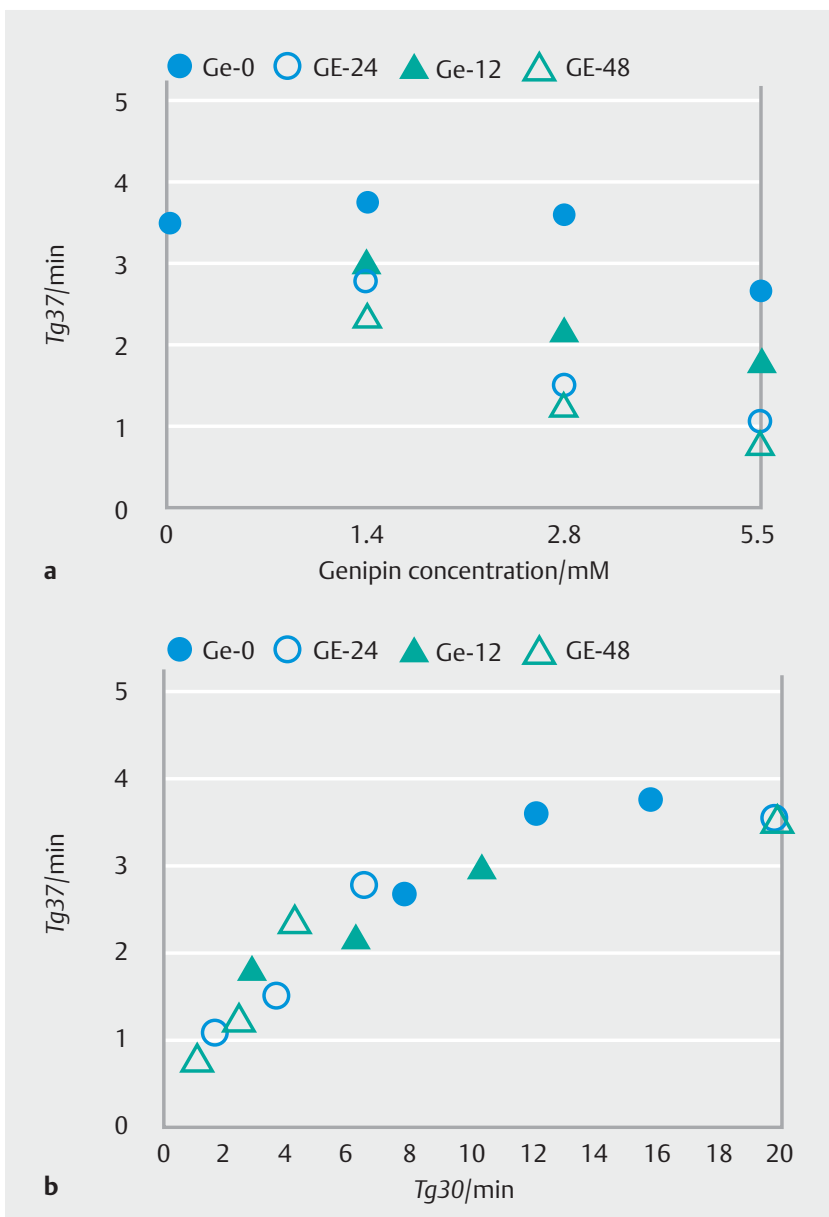

- Fig. 2 a Effects of genipin concentrations on $\mathrm{Tg} 37$ of the PSC/Ge sols containing various Ge samples. b Plots of $T g 37$ against $T g 30$ of the PSC/Ge sols containing various Ge samples at concentrations $0-5.5 \mathrm{mM}$.

significantly higher than those of NS $(80.8 \pm 5.6 \%)$ and DW $(79.3 \pm 12.5 \%)$ at 15 minutes after injection. At 30 minutes after injection, the value for PSC/Ge sol (100 $\pm 0 \%$ ) was significantly higher than those for NS (69.2 $\pm 5.6 \%)$, DW (67.0 \pm $12.9 \%)$, and $\mathrm{SH}(71.2 \pm 5.0 \%)$. At 45 and 60 minutes after injection, PSC/Ge sol exhibited results similar to those seen $30 \mathrm{~min}$ utes after injection. PSC/Ge sol (100 $\pm 0 \%)$ presented a significantly higher value than NS (61.7 $\pm 11.2 \%)$, DW (67.0 $\pm 12.9 \%)$, and $\mathrm{SH}(66.8 \pm 7.1 \%)$ at 45 minutes after injection. Finally, the value for PSC/Ge sol (100 $\pm 0.0 \%)$ was significantly higher than those for NS $(61.7 \pm 11.2 \%)$, DW $(58.3 \pm 11.8 \%)$ and SH $(61.8 \pm$ $8.6 \%)$ at 60 minutes after injection. Mean retention rates for mucosal elevation of PSC/Ge sol and PSC sol at 60 minutes after injection did not exhibit a significant difference $(P=0.08)$ ( $>$ Fig.6b). As shown in $>$ Fig. 7, mucosal elevation of NS was completely extinguished at 60 minutes after injection. In contrast, we macroscopically observed that PSC/Ge sol formed a gel in the submucosa immediately after injection. Furthermore, PSC/Ge sol retained its formation and was fixed in the submucosa for at least 60 minutes. 


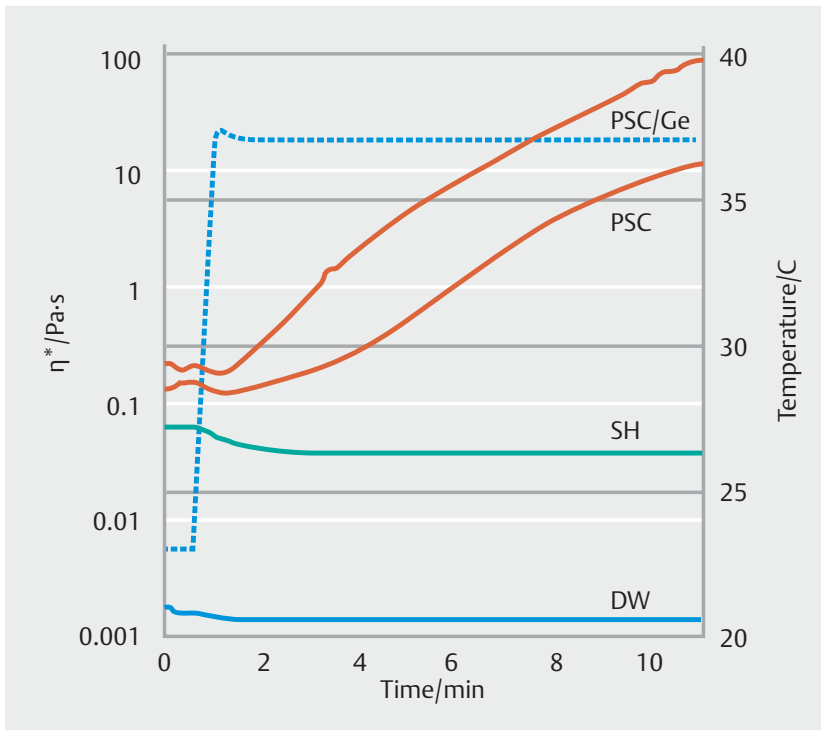

- Fig. 3 Viscosity change of various submucosal injection materials. Solid lines indicate complex viscosity $\left(\eta^{*}\right)$. Dotted line indicates set temperature of a rheometer. Normal saline (NS) was not tested.

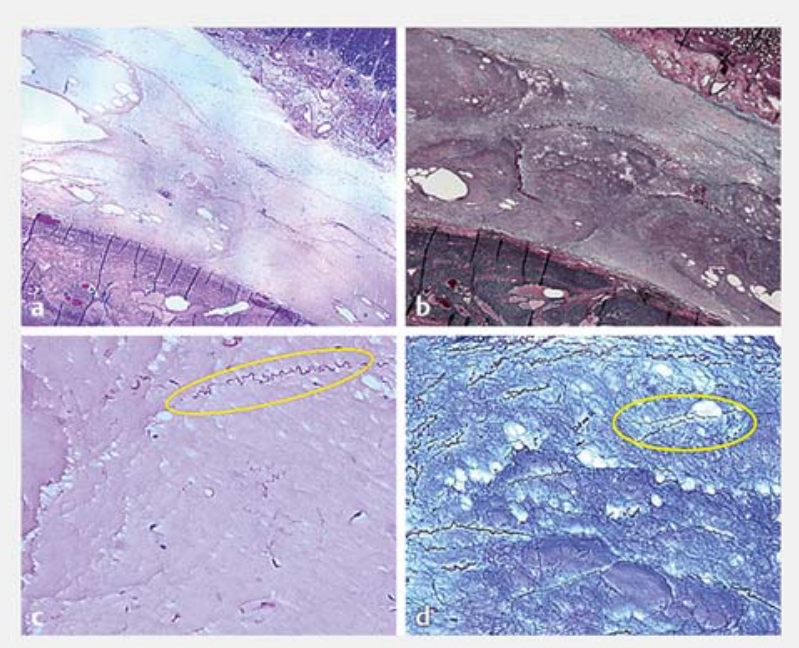

- Fig. 4 The PSC gel in SM layer which was observed microscopically. a HE staining $(\times 20)$, b HE staining $(\times 100)$, c Sliver impregnation staining $(\times 20)$, d Sliver impregnation staining $(\times 100)$. $\mathrm{PCS} / \mathrm{Ge}$ is present fully in $\mathbf{b}$ and $\mathbf{d}$ except reticular fiber; the spiral structure (surrounded yellow circle).

\section{Discussion}

In this study, we addressed PSC, PB, and genipin and determined the proper conditions for these substances according to previous reports $[14,15]$. The optimal conditions maintain fluidity in an endoscope and an endoscopic catheter and result in rapid gelation in the submucosa. Our preliminary examinations showed that the channel temperature of an endoscope inserted into the porcine stomach via the esophagus was $30^{\circ} \mathrm{C}$; therefore, $\operatorname{Tg} 30$ indicates the minimum retention time of fluid-

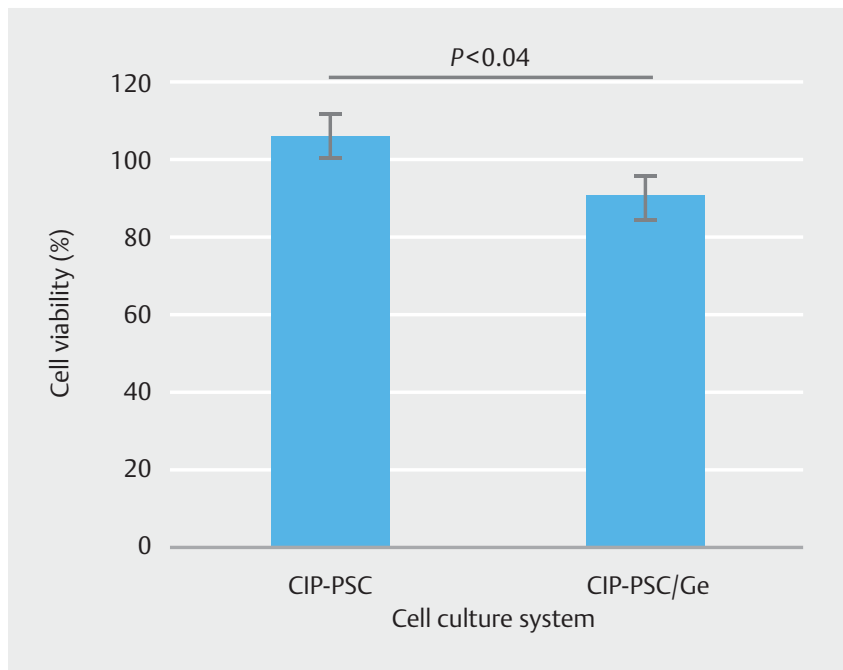

Fig. 5 Results of cell viability tests. Data are presented as mean $\pm \operatorname{SD}(n=5)$.

ity when the sol is introduced through the scope channel. In determining the concentration of $\mathrm{NaCl}$, we emphasized that sufficient fluidity should be maintained in an endoscopic catheter, rather than focusing on the gelation time, and we adopted a concentration of $280 \mathrm{mMol}$ because it is an integer multiple of the concentration of NS. Regarding determination of the warming time and concentration of genipin, we prioritized initiation of gelation within approximately 1 minutes at body temperature. Finally, we set the conditions of PSC/Ge sol as follows: PSC/2.8 mM Ge-24 sol in PB[50-280]. As shown in > Fig. 3, PSC/Ge sol initiated gelation immediately after reaching $37^{\circ} \mathrm{C}$, and the mechanical property increased linearly, sustainably, and rapidly over time. In addition, the mechanical property of $\mathrm{PSC} / \mathrm{Ge}$ sol was greater than that of PSC sol throughout this examination because of the crosslinking effect of genipin.

We set the conditions of collagen, genipin, and PB to realize rapid gelation at $37^{\circ} \mathrm{C}$, similar to body temperature, and gelation was generally not observed at the temperature in the catheter lumen in this study. However, we had to prove that PSC/Ge sol integrated with the submucosa. Agents that only extend into the submucosa vertically are unsuitable as submucosal injection material for endoscopic procedures. If submucosal injection material extends into the submucosa vertically, it could lead to physical damage to surrounding tissue. Therefore, we conducted a pathological evaluation to confirm the integration of the collagen gel into the submucosa. We observed that PSC/ Ge sol was present between the reticular fibers under silver impregnation staining, which indicated that PSC/Ge sol integrated with the SN and did not extend into the surrounding tissue vertically. Thus, PSC/Ge sol was suitable as a submucosal injection material.

Collagen has been used for clinical applications, and its safety in living tissue has been verified [16-19]. In addition, genipin has long been used as a Kampo medicine, and it is reported to be safer than other crosslinking agents [14]. However, we performed an in vitro examination again to evaluate the cyto- 


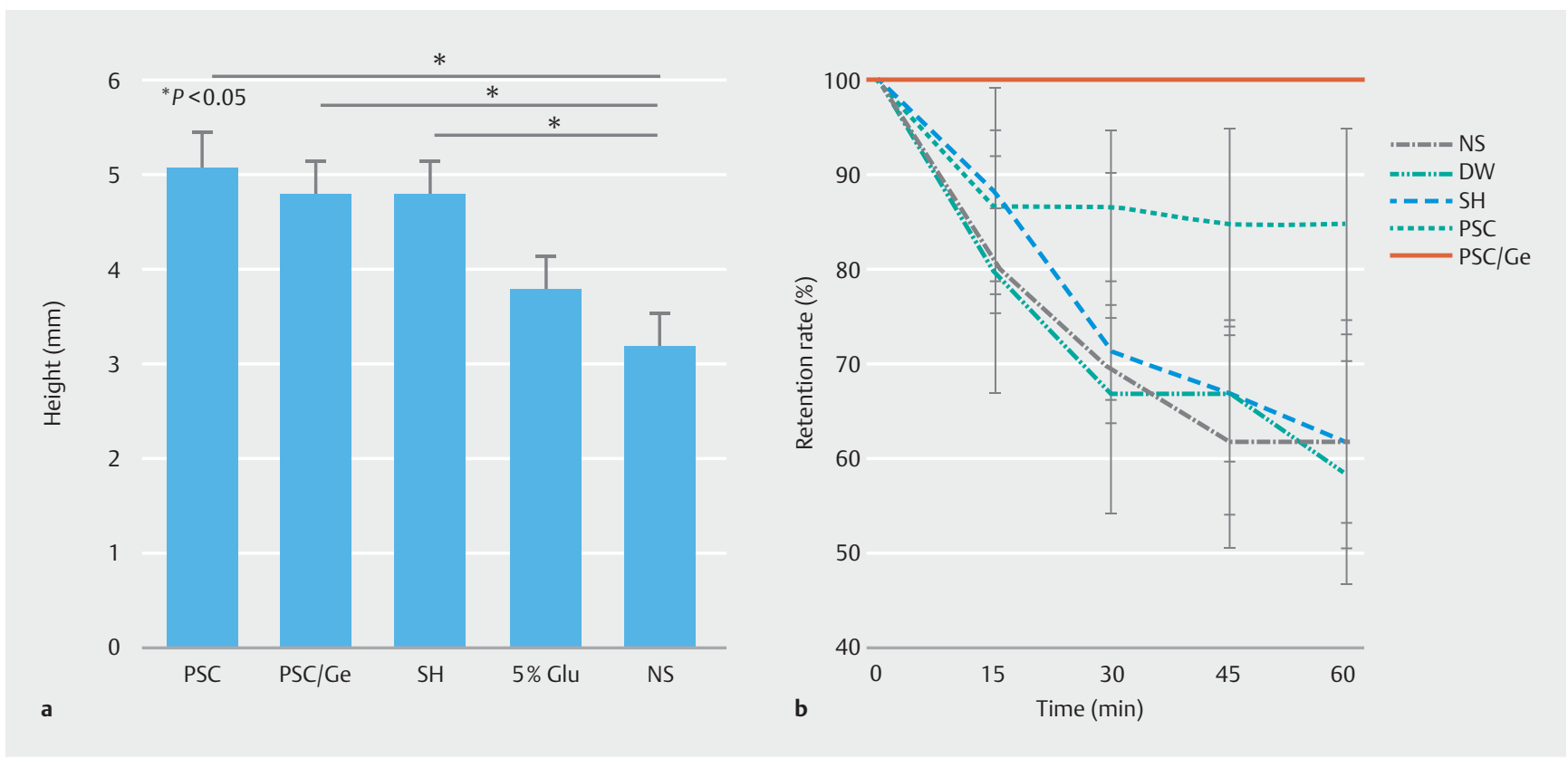

- Fig. 6 a Comparison of SM elevation after immediately injection. b Transitive graph of retention rate of SM elevation until after 60 minutes after injection.

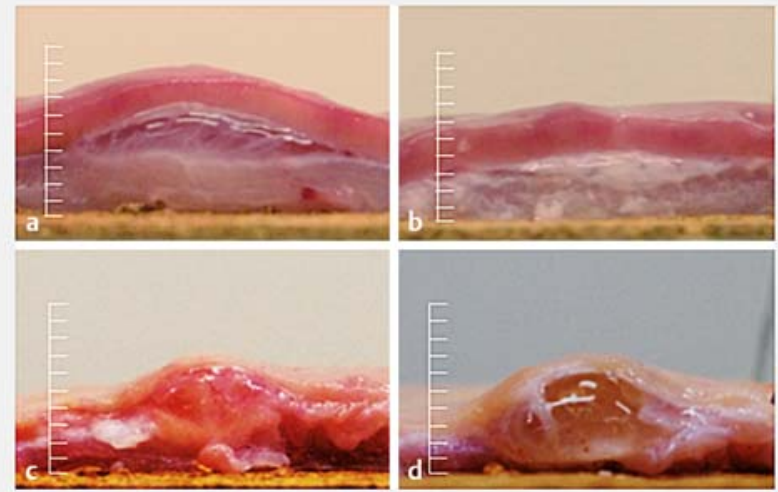

- Fig. 7 Sectional view of porcine stomachs. a just after injection of NS. b Sixty minutes after injection of NS. c Just after injection of the PSC/Ge sol. d Sixty minutes after injection of the PSC/Ge sol.

toxicity of genipin for use as a novel submucosal injection material. In this study, we modified the cell culture system using acid-solubilized collagen in CIPs that mimicked the submucosa to evaluate the cytotoxicity of cells present in the submucosa. This study revealed that the cytotoxicity of PSC/Ge sol was higher than that of PSC sol under both methods. However, we thought that this result was acceptable, being the result of an in vitro study, and should be comprehensively considered based on the results of an in vivo study for final clinical application.

Mean heights of the mucosal elevation of PSC/Ge sol and PSC sol immediately after injection were significantly higher than that of NS; mean retention rates of the mucosal elevation of PSC/Ge sol and PSC sol at 60 minutes after injection were sig- nificantly higher than those of SH, DW and NS. These results indicate that PSC/Ge sol and PSC sol represent good submucosal injection materials. Although there was no significant difference between PSC/Ge sol and PSC sol when retention rates of mucosal elevation were compared, PSC/Ge sol exhibited a higher storage modulus than that of PSC sol in rheometer testing. Genipin increased the mechanical properties of collagen by crosslinking collagen fibers, which could lead to a $100 \%$ retention rate of PSC/Ge sol at $60 \mathrm{~min}$. Based on these results, we concluded that PSC/Ge sol is better than PSC sol as a submucosal injection material.

In addition, PSC/Ge sol costs approximately 11 USD (calculated at 100 yen per dollar) for $20 \mathrm{~mL}$, as calculated from the material cost. In contrast, SH costs approximately 42.5 USD for $20 \mathrm{~mL}$, as calculated from the material cost in Japan. Thus, PSC/ $\mathrm{Ge}$ is approximately one-quarter the price of $\mathrm{SH}$ in terms of material cost. Furthermore, it may be possible to perform endoscopic intervention using less PSC/Ge sol than SH because it remains in the submucosa for a long period according to study 4. Therefore, it could have a good effect on the medical economy.

In this study, we investigated the physical properties of each material quantitatively with a rheometer. Although there have been many reports in which the retention rate of the mucosal elevation has been configured as a barometer of physical properties $[3,4,8]$, no studies have evaluated physical properties with a rheometer. Although most studies evaluate safety through in vivo experiments, no reports exist of evaluation of cytotoxicity of submucosal injection material through in vitro experiments. We adopted precise, objective measurement methods in this study. Therefore, the results of our study are very reliable compared to past reports. 
There are some limitations to this study. First, this was an in vitro study. We investigated only the basal properties of PSC/Ge sol and PSC sol. An in vivo study needs to be conducted to validate the safety and usability of these products using a live porcine model for clinical application. Second, we did not evaluate the retention rate of the mucosal elevation after 60 minutes post-injection. Endoscopic resection procedure times are currently seldom greater than 1 hour, although progress in endoscopic techniques and devices has enabled more difficult and challenging cases to be addressed. Therefore, a long-term dynamic analysis of PSC/Ge sol should be performed over 1 hour.

\section{Conclusion}

In conclusion, we suggest that the basal properties of collagen and genipin make them suitable as a submucosal injection material for endoscopic resection. PSC/Ge sol integrated with the submucosa macroscopically and showed relatively low cytotoxicity. Moreover, PSC/Ge sol exhibited a good retention rate of the mucosal elevation compared to existing submucosal injection material by crosslinking with genipin. Therefore, PSC/ Ge sol is considered to be a good submucosal injection material. In vivo studies to evaluate the safety and usability of PSC/Ge sol will be necessary in the near future.

\section{Competing interests}

None

\section{References}

[1] Soetikno RM, Gotoda T, Nakanishi Y et al. Endoscopic mucosal resection. Gastrointest Endosc 2003; 57: 567-579

[2] Antillon MR, Bartalos CR, Miller ML et al. En bloc endoscopic submucosal dissection of a $14-\mathrm{cm}$ laterally spreading adenoma of the rectum with involvement to the anal canal: expanding the frontiers of endoscopic surgery (with video). Gastrointest Endosc 2008; 67: 332 337

[3] Liu W, Zhao M, Liu W et al. A feasibility study of a thermally sensitive elastin-like polypeptide for submucosal injection application in endoscopic resection in 3 animal models. Gastrointest Endosc 2015; 82: $944-952$

[4] Fujishiro M, Yahagi N, Kashimura K et al. Comparison of various submucosal injection solutions for maintaining mucosal elevation during endoscopic mucosal resection. Endoscopy 2004; 36: 579-583
[5] Hikichi T, Yamasaki M, Watanabe K et al. Gastric endoscopic submucosal dissection using sodium carboxymethylcellulose as a new injection substance. Fukushima J Med Sci 2016; 62: 43 - 50

[6] Pioche M, Lepilliez V, Deprez P et al. High pressure jet injection of viscous solutions for endoscopic submucosal dissection (ESD): first clinical experience. Endosc Int Open 2015; 3: E368-372

[7] Wen W, Shi C, Shi Y et al. A pilot animal and clinical study of autologous blood solution compared with normal saline for use as an endoscopic submucosal cushion. Exper Therap Med 2012; 4: 419-424

[8] Akagi T, Yasuda K, Tajima M et al. Sodium alginate as an ideal submucosal injection material for endoscopic submucosal resection: preliminary experimental and clinical study. Gastrointest Endosc 2011; 74: 1026 - 1032

[9] Uraoka T, Kawahara Y, Ohara $\mathrm{N}$ et al. Carbon dioxide submucosal injection cushion: an innovative technique in endoscopic submucosal dissection. Digest Endosc 2011; 23: 5-9

[10] Liu W, Wang M, Zhao L et al. Thermo-sensitive isopentane aerification for mucosal lift during endoscopic resection in animal models (with video). Gastrointest Endosc 2017; 86: 1168-1175 e1163

[11] Sundararaghavan HG, Monteiro GA, Lapin NA et al. Genipin-induced changes in collagen gels: correlation of mechanical properties to fluorescence. J Biomed Mater Res Part A 2008; 87: 308-320

[12] Yunoki S, Ohyabu Y, Hatayama H. Temperature-responsive gelation of type I collagen solutions involving fibril formation and genipin crosslinking as a potential injectable hydrogel. Int J Biomater 2013; 2013: 620765

[13] Narita T, Yunoki S, Ohyabu Y et al. In situ gelation properties of a collagen-genipin sol with a potential for the treatment of gastrointestinal ulcers. Med Devices (Auckl) 2016; 9: 429-439

[14] Sung HW, Huang RN, Huang LL et al. Feasibility study of a natural crosslinking reagent for biological tissue fixation. J Biomed Mat Res 1998; 42: 560-567

[15] Yunoki S, Hatayama $\mathrm{H}$, Ebisawa $\mathrm{M}$ et al. A novel fabrication method to create a thick collagen bundle composed of uniaxially aligned fibrils: an essential technology for the development of artificial tendon/ligament matrices. J Biomed Mat Res Part A 2015; 103: 3054- 3065

[16] Ruszczak Z, Schwartz RA. Collagen uses in dermatology - an update. Dermatology (Basel, Switzerland) 1999; 199: $285-289$

[17] Charriere G, Bejot M, Schnitzler L et al. Reactions to a bovine collagen implant. Clinical immunologic study in 705 patients. J Am Acad Dermatol 1989; 21: $1203-1208$

[18] Macaya D, Spector M. Injectable collagen-genipin gel for the treatment of spinal cord injury: in vitro studies. Advanced Functional Materials 2011; $21: 4788-4797$

[19] Yen Chang C-CT, Huang-Chien Liang, Hsing-Wen Sung. In vivo evaluation of cellular an acellular bovine pericardia fixed with a naturally occurring crosslinking agent (genipin). Biomaterials 2002; 23: 2447 2457 